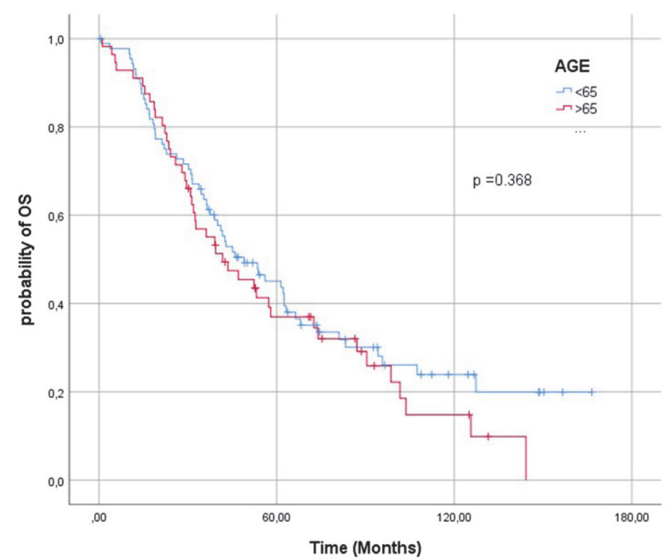

Kaplan Meier Curves. Overall Survival in advanced ovarian cáncer after debulking surgery

Abstract 49 Figure 1 Kaplan Meier curves. Overall survival in advance ovarian cancer after bebulking surgery

stage. Charlson comorbidity index and tumor grade were higher among aged patients. The proportion of PDS and the surgical complexity score did not show statiscally signficative differences, as well as the rate of major postoperative complications or length of stay.

Patients over 65 years had optimal cytorreduction in $78.3 \%$, the progression free survival was 19.1 months and overall survival was the 48.7 months compared with patients up to 65 years, which had $87.1 \%$ of optimal debulking surgery, 24.6 months of DFS and 52.7 months. None of these outcomes revealed any statistical significant difference between groups.

Conclusion* The survival outcomes in elderly ovarian cancer patients are the same as younger patients. The age should not be the main factor to decide the upfront treatment of AEOC.

\section{PATIENT REPORTED OUTCOME IN PLATINUM- RESISTANT RECURRENT OVARIAN CANCER AND METASTATIC BREAST CANCER TREATED WITH METRONOMIC CHEMOTHERAPY}

S Krajnak*, M Battista, K Almstedt, K Anic, AS Heimes, V Linz, R Schwab, A Hasenburg, M Schmidt. University Medical Centre of the Johannes Gutenberg University Mainz, Department of Gynaecology and Obstetrics, Mainz, Germany

\subsection{6/ijgc-2021-ESG0.336}

Introduction/Background* In the treatment of both platinumresistant recurrent ovarian cancer (ROC) and metastatic breast cancer (MBC), symptom control and maintenance of quality of life (QoL) play a crucial role. In the advanced stage of disease, metronomic chemotherapy (MCT) may be a favourable treatment option. The aim of this study is to assess the QoL of heavily pretreated patients with ROC and MBC treated with MCT.

Methodology PROmetronomic, FoR.UM 19-02193, is a monocentric, open-label, single-arm observational study to assess health-related patient-reported outcome data in ROC and MBC patients treated with MCT (cyclophosphamide $50 \mathrm{mg}$ p. o. daily \pm methotrexate $2.5 \mathrm{mg}$ p.o. every other day). QoL data are evaluated using European Organisation for Research and Treatment of Cancer (EORTC) QLQ-C30 version 3.0, EORTC QLQ-OV28 version 1.0 (ROC)/EORTC QLQ-BR23

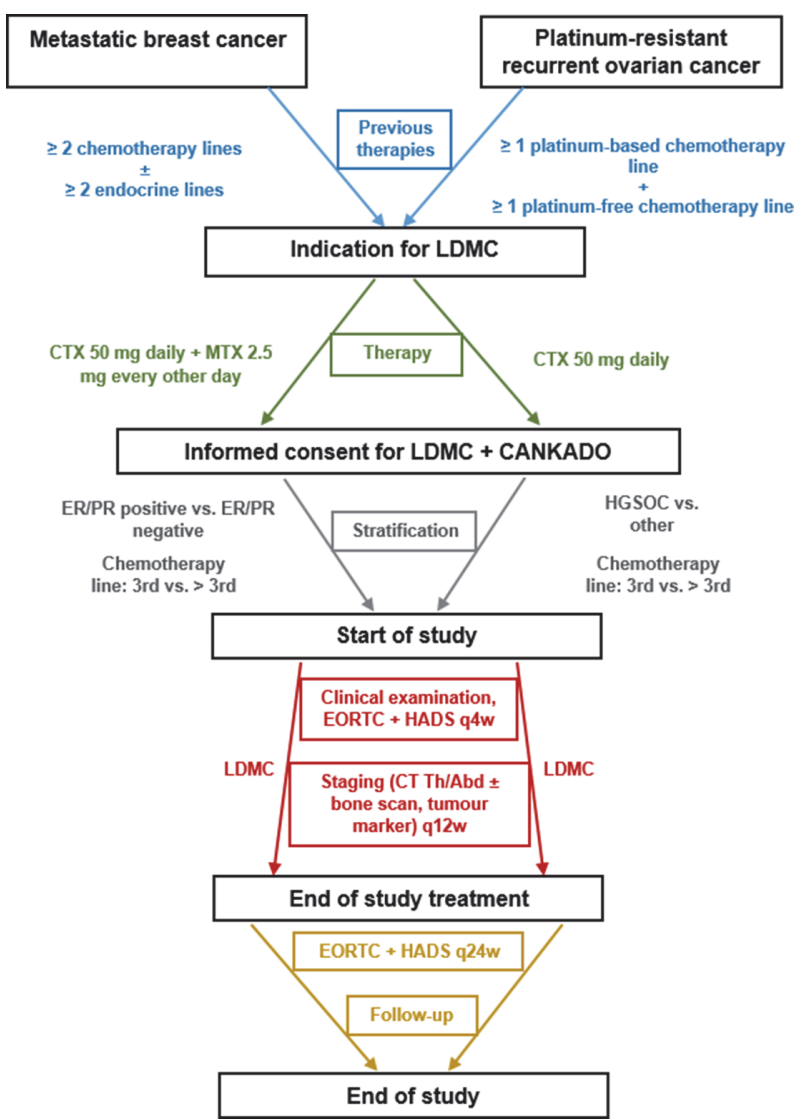

Abstract 70 Figure 1

version $1.0(\mathrm{MBC})$, and Hospital anxiety and depression scale (HADS-D) questionnaires via an internet-based therapy support system CANKADO. Patients previously treated with at least 1 line of platinum-based and 1 platinum-free chemotherapy (ROC)/at least 2 lines of endocrine therapy (for hormone receptor-positive cancer) and at least 2 lines of chemotherapy (MBC) are included. Secondary endpoints are disease control rate at 12 and 24 weeks, duration of response, progressionfree survival and overall survival. Assessment of safety and tolerability is conducted according to the National Cancer Institute Common Terminology Criteria for Adverse Events (NCI CTCAE) version 5.0. As part of the translational research approach, potentially relevant anti-angiogenic and immunomodulatory biomarkers are being investigated.

Result(s)* Until 2021-05-01, 4 ROC and 3 MBC patients have been enrolled. It is planned to include a total of 65 patients until 08/2023.

Conclusion* Potentially toxic chemotherapy is often required to achieve disease control in patients with metastatic cancer. However, well-being and personal preferences must not be neglected. MCT could provide an efficacious treatment option with limited toxicities and positive impact on QoL.

\section{CYTOLOGICAL SAMPLES FOR DETECTION OF BRCA $1 / 2$ MUTATIONS IN PATIENTS WITH HIGH GRADE SEROUS OVARIAN CANCER}

E Skof*, S Novaković, V Stegel, S Miceska, M Krajc, S Bebar, V Kloboves-Prevodnik, $\checkmark$ Setrajcic Dragos, A Blatnik. Institute of Oncology Ljubljana, Slovenia

10.1136/ijgc-2021-ESG0.337 